\title{
ON THE THEORY OF LINEAR PARTIAL DIFFERENTIAL OPERATORS WITH ANALYTIC COEFFICIENTS
}

\author{
BY \\ FRANÇOIS TREVES
}

Introduction. Consider a linear partial differential operator of order $m, \boldsymbol{P}$, having coefficients defined and analytic in some open subset $\Omega$ of $\boldsymbol{R}^{n+1}(n \geqq 0)$, and let $S$ be a piece of analytic hypersurface in $\Omega$, noncharacteristic with respect to $\boldsymbol{P}$ at some point $x^{0}$ (we denote by $\partial / \partial \nu$ the differentiation in the direction of the normal to $S$ ). The present article is concerned with the Cauchy problem:

(I) $\boldsymbol{P} u=f$ in some open neighborhood $U$ of $x^{0}$;

(II) $(\partial / \partial \nu)^{k} u=g_{k}$ on $S \cap U(k=0, \ldots, m-1)$.

The main result (see $\$ 4$ ) can be roughly stated as follows: it is possible to determine the neighborhood $U$ so that, for all data fand $g_{k}(0 \leqq k \leqq m-1)$ belonging to a suitable class of "ultradistributions", there is, in the same class, a unique solution $u$ to (I)-(II). To describe the class of ultradistributions alluded to here, is not difficult. They form a one-parameter family of ultradistributions spaces (the parameter is real and denoted by $s$ ). One must first perform a special change of variables (see $\S 3$ ) by which the set $U$ is transformed into the $\operatorname{strip}\left\{(x, t) \in \boldsymbol{R}^{n+1} ;|t|<\eta, \eta>0\right\}$, and the hypersurface $S$ into the hyperplane $t=0$. Then we may say the following: the right-hand side $f$ and the solution $u$ are functions of $t$ with values in the spaces of ultradistributions with respect to the variables $x$, which we introduce under the notation $K^{s}$; the Cauchy data $g_{k}$ are members of such $K^{s}$ spaces. What is the space $K^{s}$ ? A member $v$ of $K^{s}$ is, by definition, the Fourier transform of a function $\hat{v}(\xi)$ in $\boldsymbol{R}_{n}$ which is square-integrable with respect to the measure $e^{-2 s|\xi|} d \xi$. For $s<0$, the elements of $K^{s}$ are analytic functions, holomorphically extendable to slabs $|\operatorname{Im} x|$ $<-s$ of the complex space. In this case, the main theorem reduces more or less to the classical Cauchy-Kovalevska theorem, of which it provides what I think is a new proof (in $\S 5$ ). For $s<0$, the class $K^{s}$ is quite large: it contains various types of distributions in $\boldsymbol{R}^{n}$, among which are all the ones that have compact support. In particular, the main theorem implies that the equation $\boldsymbol{P} u=f$, with $f$ say a continuous function with compact support in $U$, is solvable (in $U$ ). Of course, the solution is not going to be a distribution, a fortiori not a function-at least in general. At any event, this very weak solvability result justifies a remark made at the beginning of the article by Nirenberg-Treves [2] (a remark which has been slightly mysterious until now, to the authors of the article among others $)\left({ }^{1}\right)$. In this

Received by the editors July 14, 1967.

(1) See p. 331, line 13. 
same case $s<0$, the uniqueness of the solution $u$ to the Cauchy problem (I)-(II) yields at once the classical Holmgren's theorem (this is proved in §5).

Exploitation of the properties of the spaces $K^{s}$ is made possible by a very general result of the Cauchy-Kovalevska type, stated and proved in $\S 0$, which applies to a certain type of "operational differential equation"

$$
d \mathbf{u} / d t-B(t) \wedge \mathbf{u}=\mathbf{f} .
$$

In this article, this general result is applied to the spaces $K^{s}$ by choosing, as the operator $\wedge$, the square root of $1-\Delta\left(\Delta:\right.$ the Laplace operator in $\left.R^{n}\right)$. But other choices are possible, adapted to different problems. It seems to me that this theorem (whose proof is quite easy) should have rather far ranging applications, besides the one considered here, applications where analyticity of the coefficients and the operator $(1-\Delta)^{1 / 2}$ are replaced by different smoothness properties and suitably adapted operators (for instance, smoothness could mean to belong to the $d$ th Gevrey class and $\wedge$ be the operator $(1-\Delta)^{1 / 2 d}$-with perhaps stricter requirements on the linear partial differential equation under study).

Notations. PDE: partial differential equation,

$\boldsymbol{R}^{n}: n$-dimensional Euclidean space, $\boldsymbol{R}_{n}$ : its dual;

$C^{n}$ : $n$-dimensional complex space;

$x=\left(x_{1}, \ldots, x_{n}\right), y=\left(y_{1}, \ldots, y_{n}\right)$ : variables in $\boldsymbol{R}^{n}$;

$\xi=\left(\xi_{1}, \ldots, \xi_{n}\right), \eta=\left(\eta_{1}, \ldots, \eta_{n}\right)$ : variables in $\boldsymbol{R}_{n}$;

$D_{x_{j}}=\partial / \partial_{j}(1 \leqq j \leqq n), D_{t}=\partial / \partial t$ or $d / d t ; u^{\prime}(t)=D_{t} u(t)$;

$L^{2}$ : space of square-integrable functions in $\boldsymbol{R}^{n}$ or in $\boldsymbol{R}_{n}$.

$\mathscr{F}$ and $\mathscr{F}^{-1}$ : Fourier transformation and its inverse:

$$
(\mathscr{F} u)(\xi)=\int_{R^{n}} e^{-i\langle x, \xi\rangle} u(x) d x, \quad i=\sqrt{ }-1 .
$$

0. A general Cauchy-Kovalevska theorem. In this preliminary section, we present a generalization of the Cauchy-Kovalevska theorem. All existence and/or uniqueness results of this article are based on this theorem. In particular, we shall see in later sections that the classical Cauchy-Kovalevska theorem follows from it. The proof of the generalized version of the Cauchy-Kovalevska theorem is similar to the proof of its classical counterpart, given in Hörmander [1, Theorem 5.1.1], and, as a matter of fact, is somewhat simpler $\left({ }^{2}\right)$.

The basic ingredients are, first of all, a one-parameter family of complex Banach spaces $E_{s}\left(s\right.$ real), with norm \|\|$_{s}$, which, for simplicity, we assume to be contained in some "big" vector space. We make the following assumption:

(0.1) if $s \geqq s^{\prime}$, we have a continuous injection $E_{s} \rightarrow E_{s^{\prime}}$ with norm $\leqq 1$.

( $\left.{ }^{2}\right)$ Added in proof. After the present article was submitted for publication, I have learned that this abstract Cauchy-Kovalevska theorem has been stated, proved and applied by $\mathbf{L}$. V. Ovsyannikov in 1965 (see [3]) and, as a matter of fact, even at an earlier date, ca. 1958, by Russian mathematicians of Gelfand's school. For further "exploitation" of Ovsyannikov's theorem, see [4]. 
The union of the spaces $E_{s}$ will be denoted by $E_{-\infty}$.

Next basic ingredient: a linear operator $\wedge: E_{-\infty} \rightarrow E_{-\infty}$ about which the following hypothesis is made:

(0.2) if $s>s^{\prime}, \bigwedge$ is a bounded linear map $E_{s} \rightarrow E_{s^{\prime}}$ with norm $\leqq e^{-1}\left(s-s^{\prime}\right)^{-1}$.

Of course, the factor $e^{-1}$ has no special importance. Only, it will simplify some forthcoming formulas and actually is present in all the applications we have in mind.

The statements and the reasonings which are forthcoming are relative to functions valued in the spaces $E_{s}$. They will be functions of a variable $t$ which can be either real or complex. We shall also consider operator-valued functions of $t$. Thus, we introduce a linear operator $B(t)$, depending on $t$ and acting on a certain linear subspace of $E_{-\infty}$. More precisely, let $\rho$ and $\sigma$ be two numbers $>0$. We assume:

(0.3) For all real numbers $s,|s| \leqq \sigma$, and all $t,|t| \leqq \rho, B(t)$ is a bounded linear operator of $E_{s}$ into itself, with norm bounded by a number $\tau \geqq 0$ independent of $s$ and $t$.

We shall also need a smoothness property of $B(t)$ with respect to $t$; it will be the following one:

(0.4) Let $s$ be any real number, $|s| \leqq \sigma$. If $\mathbf{v}(t)$ is a continuous map $\{t ;|t| \leqq \rho\} \rightarrow E_{s}$, the same is true of $B(t) v(t)$. When $t$ is complex, if furthermore $\mathbf{v}(t)$ is a holomorphic map $\{t ;|t|<\rho\} \rightarrow E_{s}$, the same is true of $B(t) \mathbf{v}(t)$.

Then, the generalized Cauchy-Kovalevska theorem will apply to the equation

$$
\mathbf{u}^{\prime}(t)-B(t) \wedge \mathbf{u}(t)=\mathbf{f}(t)
$$

with initial datum

$$
\mathbf{u}(0)=\mathbf{u}_{0}
$$

As usual, $\mathbf{u}^{\prime}(t)$ stands for the derivative of $\mathbf{u}(t)$ with respect to $t$. We must also say what kind of data $\mathbf{f}(t), \mathbf{u}_{0}$ we are willing to consider, and where equation $(0.5)$ should be valid.

We choose arbitrarily a real number $s_{0},\left|s_{0}\right| \leqq \sigma$. We require that

(0.7) $\mathbf{u}_{0} \in E_{s_{0}}$ and $\bigwedge \mathrm{u}_{0} \in E_{s_{0}}$,

(0.8) $\mathbf{f}(t)$ is a continuous map $\{t ;|t| \leqq \rho\} \rightarrow E_{s_{0}}$; if $t$ is complex, $\mathbf{f}(t)$ is also holomorphic for $|t|<\rho$ with values in $E_{s_{0}}$.

We may now state the announced result (when $t$ is complex, the expression "continuously differentiable" in the statement must be understood as meaning "holomorphic"):

THEOREM 0.1. Under the preceding hypotheses, there is a unique function $\mathbf{u}(t)$ which for some number $\varepsilon, 0<\varepsilon<\rho$, and for some real number $s<s_{0},|s|<\sigma$, is a continuously differentiable function of $t,|t|<\varepsilon$, with values in $E_{s}$, and verifies (0.5) for $|t|<\varepsilon$, and (0.6).

This can be complemented by the following statement:

THEOREM 0.2. Let $s$ be a real number such that $s<s_{0},|s|<\sigma$ and $s_{0}-s \leqq \rho \tau$. 
The function $\mathbf{u}(t)$ of Theorem 0.1 has the following properties:

(0.9) $\mathbf{u}(t)$ is a continuously differentiable function of $t,|t|<\tau^{-1}\left(s_{0}-s\right)$, with values in $E_{s}$;

(0.10) we have, for all $t,|t|<\tau^{-1}\left(s_{0}-s\right)$,

$$
\begin{aligned}
\|\mathbf{u}(t)\|_{s} & \leqq\left\|\mathbf{u}_{0}\right\|_{s}+\frac{C}{\tau}\left(s_{0}-s\right)\left|\log \left(1-\frac{\tau|t|}{s_{0}-s}\right)\right| \\
\|\wedge \mathbf{u}(t)\|_{s} & \leqq\left\|\wedge \mathbf{u}_{0}\right\|_{s}+C|t|\left\{\left(s_{0}-s\right)-\tau|t|\right\}^{-1} \\
\left\|\mathbf{u}^{\prime}(t)\right\|_{s} & \leqq C\left(1-\tau|t| /\left(s_{0}-s\right)\right)^{-1}
\end{aligned}
$$

where

$$
C=\tau\left\|\wedge \mathbf{u}_{0}\right\|_{s_{0}}+\sup _{|t| \leq \rho} \mathbf{f}\|(t)\|_{s_{0}}
$$

Proof. We make the substitution $\mathbf{u}=\mathbf{v}+\mathbf{u}_{0}$ in (0.5)-(0.6), which become:

$$
\mathbf{v}^{\prime}-B \wedge \mathbf{v}=\mathbf{g}, \quad \mathbf{v}(0)=0,
$$

with $\mathbf{g}(t)=\mathbf{f}(t)+B(t) \wedge \mathbf{u}_{0}$. Because of our hypotheses, $\mathbf{g}$ has the same smoothness properties as $\mathbf{f}$. We shall prove the existence of the solution $\mathbf{v}$ by iteration, more precisely, by using the recursion formula

$$
\mathbf{v}_{k+1}^{\prime}=B \wedge \mathbf{v}_{k}+\mathbf{g}, \quad \mathbf{v}_{k}(0)=0 \quad(k=0,1, \ldots) .
$$

We start the recursion with $\mathbf{v}_{0} \equiv 0$, and we set $\mathbf{w}_{k}=\mathbf{v}_{k+1}-\mathbf{v}_{k}$. We get

$$
\begin{gathered}
\mathbf{w}_{0}^{\prime}=\mathbf{g}, \quad \mathbf{w}_{0}(0)=0, \\
\mathbf{w}_{k+1}^{\prime}=B \wedge \mathbf{w}_{k}, \quad \mathbf{w}_{k+1}(0)=0 \quad(k=0,1, \ldots) .
\end{gathered}
$$

We are going to prove that, for all $k=0,1, \ldots$, all $s<s_{0},|s| \leqq \sigma$, all $t,|t| \leqq \rho$,

$$
\left\|\mathbf{w}_{k}^{\prime}(t)\right\|_{s} \leqq C \tau^{k}|t|^{k} d^{-k},
$$

where $C$ is the constant in Theorem 0.2 and $d=s_{0}-s$. First of all, $(0.18)$ holds for $k=0$, because, in view of $(0.3), \sup _{|t| \leqq \rho}\|\mathbf{g}(t)\|_{s} \leqq C$. Suppose then that $(0.18)$ holds for a given $k \geqq 0$. By integration with respect to $t$, we obtain

$$
\left\|\mathbf{w}_{k}(t)\right\|_{s} \leqq C \tau^{k} \frac{|t|^{k+1}}{k+1} d^{-k} .
$$

We apply this with $s+\varepsilon, 0 \leqq \varepsilon \leqq d$, substituted for $s$, and we exploit, at this point, hypothesis $(0.2)$. We obtain

$$
\left\|\wedge \mathbf{w}_{k}(t)\right\|_{s} \leqq C e^{-1} \tau^{k} \frac{|t|^{k+1}}{k+1} \varepsilon^{-1}(d-\varepsilon)^{-k} .
$$

We choose $\varepsilon=d /(k+1)$; $(0.20)$ becomes

$$
\left\|\wedge \mathbf{w}_{k}(t)\right\|_{s} \leqq C e^{-1}(1+1 / k)^{k} \tau^{k}|t|^{k+1} d^{-(k+1)} .
$$

We combine (0.21) with (0.17), and we take (0.3) into account, which yields at once 
(0.18) with $k$ replaced by $k+1$. Setting $\mathbf{u}=\mathbf{u}_{0}+\sum_{k=0}^{+\infty} \mathbf{w}_{k}$ gives all the desired results. In particular, (0.18) implies (0.13), (0.19) implies (0.11) and (0.21) implies (0.12).

Finally, we must prove the uniqueness of the solution $\mathbf{u}$, that is, we prove that if a continuously differentiable map

$$
\mathbf{w}(t):\{t ;|t|<\varepsilon\} \rightarrow E_{s_{0}^{\prime}}
$$

for $s_{0}^{\prime}<s_{0},\left|s_{0}^{\prime}\right|<\sigma$, satisfies

$$
\mathbf{w}^{\prime}=B \wedge \mathbf{w} \quad \text { for }|t|<\varepsilon, \quad \mathbf{w}(0)=0,
$$

it must vanish identically for $|t|<\varepsilon$. Let us set $\mathbf{g}(t)=B(t) \wedge \mathbf{w}(t)$. Obviously $\mathbf{g}$ is a continuous function of $t,|t|<\varepsilon$ (we may now restrict ourselves to the case where $t$ is real, although the reasoning is still valid when $t$ is complex, independently of holomorphy), with values in $E_{s_{0}^{\prime \prime}}$ with $s_{0}^{\prime \prime}<s_{0}^{\prime},\left|s_{0}^{\prime \prime}\right|<\sigma$. But then $(0.22)$ tells us that the sequence $\left\{\mathbf{w}_{k}\right\}$, where $\mathbf{w}_{k}=\mathbf{w}$ for all $k=0,1, \ldots$, verifies $(0.17)$, therefore also $(0.19)$ for all $k$, with $s_{0}^{\prime \prime}$ substituted for $s_{0}$ and $\varepsilon$ for $\rho$. But this means that $\mathbf{w}(t)=0$ for $|t|<\tau^{-1} d$. If $\varepsilon$ happens to be $>\tau^{-1} d$, we perform a translation and bring the origin at the points $\pm \tau^{-1} d$, and repeat the argument. After a finite number of steps, we see that $\mathrm{w}(t)=0$ for all real $t,|t|<\varepsilon$. Q.E.D.

1. Spaces $K^{s}$. We consider the following functions of $\xi \in \boldsymbol{R}_{n}$ :

$$
\begin{aligned}
h_{j}(\xi) & =\left(1+\xi_{j}^{2}\right)^{1 / 2} \quad(1 \leqq j \leqq n), \\
h(\xi) & =h_{1}(\xi)+\cdots+h_{n}(\xi) .
\end{aligned}
$$

DeFINITION 1.1. Let $s$ be a real number. We shall denote by $\hat{K}^{s}$ the space of (classes of) functions $F$ in $\boldsymbol{R}_{n}$ such that $e^{\text {sh( }(\xi)} F(\xi) \in L^{2}$.

Let $s^{\prime}$ be another real number. We shall denote by $\hat{K}^{s, s^{\prime}}$ the space of functions $F$ in $\boldsymbol{R}_{n}$ such that $h^{s^{\prime}} e^{s h} F \in L^{2}$.

Of course, $\hat{K}^{s, 0}=\hat{K}^{s}$. The spaces $\hat{K}^{s, s^{\prime}}$ carry a natural Hilbert space structure: the one carried over from $L^{2}$ via the vector space isomorphism $F \leftrightarrow h^{s^{\prime}} e^{s h} F$.

It is clear that $\mathscr{C}_{c}^{\infty}\left(\boldsymbol{R}_{n}\right)$ is a dense subspace of $\hat{K}^{s, s^{\prime}}$. The inverse Fourier transformation, $\mathscr{F}^{-1}$, is a vector space isomorphism of $\mathscr{C}_{c}^{\infty}\left(\boldsymbol{R}_{n}\right)$ onto $(\operatorname{Exp} \cap \mathscr{S})\left(\boldsymbol{R}^{n}\right)$, the space of rapidly decreasing $\mathscr{C}^{\infty}$ functions in $\boldsymbol{R}^{n}$ which can be extended to $\boldsymbol{C}^{n}$ as entire functions of exponential type. For functions $u, v \in(\operatorname{Exp} \cap \mathscr{S})$, we define the (Hilbert) norm

$$
\|u\|_{s, s^{\prime}}=(2 \pi)^{-n / 2}\left\|h^{s^{\prime}} e^{s h} \hat{u}\right\|_{L^{2}}
$$

and associated inner product

$$
(u, v)_{s, s^{\prime}}=(2 \pi)^{-n} \int h^{2 s^{\prime}}(\xi) e^{2 \operatorname{sh}(\xi)} \hat{u}(\xi) \overline{\hat{v}(\xi)} d \xi .
$$

Definition 1.2. We denote by $K^{s, s^{\prime}}$-by $K^{s}$ when $s^{\prime}=0$-the completion of the space $(\operatorname{Exp} \cap \mathscr{S})$ normed by (1.1). 
The Fourier transformation $\mathscr{F}$ is an isometry of $(\operatorname{Exp} \cap \mathscr{S})\left(\boldsymbol{R}^{n}\right)$-normed by (1.1)-onto $\mathscr{C}_{c}^{\infty}\left(\boldsymbol{R}_{n}\right)$, viewed as a subspace of the Hilbert space $\hat{R}^{s, s^{\prime}}:$ it extends as an isometry of $K^{s, s^{\prime}}$ onto $R^{s, s^{\prime}}$. One might say that the elements of $K^{s, s^{\prime}}$ are the objects whose Fourier transform belongs to $\hat{K}^{s, s^{\prime}}$ (this justifies the "roof" in the notation $\left.\hat{K}^{s . s^{\prime}}\right)$.

Proposition 1.1. When $s$ is $\geqq 0, K^{s, s^{\prime}}$ can be canonically identified with a space of tempered distributions in $\boldsymbol{R}^{n}$. When $s=0$, this is the Sobolev space $H^{s^{\prime}}$. When $s>0$, it is a space of functions which can be extended as holomorphic functions in the strip $\left\{z \in C^{n} ;\left|\operatorname{Im} z_{j}\right|<s, j=1, \ldots, n\right\}$.

Proof. When $s \geqq 0, \hat{K}^{s, s^{\prime}}$ is clearly a space of tempered distributions, i.e. a linear subspace of $\mathscr{S}^{\prime}$ provided with a locally convex topology, finer than the one induced by $\mathscr{S}^{\prime}$. Fourier transformation, which is an automorphism of $\mathscr{S}^{\prime}$, defines an isomorphism of $K^{s, s^{\prime}}$ onto another space of tempered distributions: the latter can be identified with $K^{s, s^{\prime}}$. That $K^{0, s^{\prime}}=H^{s^{\prime}}$ is obvious in view of Definition 1.1.

Suppose $s>0$. The elements of $\hat{K}^{s}$ are functions which decay very fast at infinity, hence $K^{s} \subset \mathscr{S}^{\prime}$ consists of $\mathscr{C}^{\infty}$ functions. Reasoning in the case where $n=1$, which suffices to our purpose, we see that if $p$ is an integer $\geqq 0$,

$$
\begin{aligned}
\left|D^{p} u(x)\right| & \leqq(2 \pi)^{-1} \int|\xi|^{p} e^{-s|\xi|}\left(e^{s h(\xi)}|\hat{u}(\xi)|\right) d \xi \\
& \leqq\|u\|_{s, 0}\left(2 \int_{0}^{+\infty} t^{2 p} e^{-2 s t} d t\right)^{1 / 2}=\text { const } p ! s^{-p-1 / 2}
\end{aligned}
$$

by Stirling's formula. The case of $K^{s, s^{\prime}}, s>0, s^{\prime} \neq 0$, can be dealt with similarly, or else follows from the statement (1.4) in the next proposition:

Proposition 1.2. The following facts are true:

(1.3) If $s_{1} \geqq s_{1}^{\prime}$ and $s_{2} \geqq s_{2}^{\prime}, K^{s_{1}, s_{2}} \subset K^{s_{1}^{\prime}, s_{2}^{\prime}}$ and the natural injection of the former into the latter is continuous and has norm one.

(1.4) If $s_{1}>s_{1}^{\prime}, K^{s_{1}, s_{2}} \subset K^{s_{1}^{\prime}, s_{2}^{\prime}}$ whatever be $s_{2}, s_{2}^{\prime}$.

Proposition 1.2 is trivial; the natural injections it tells us about are the canonical extensions of the identity mapping of $(\operatorname{Exp} \cap \mathscr{S})$.

We may introduce the spaces

$$
K^{+\infty}=\bigcap_{s \in R} K^{s}, \quad K^{-\infty}=\bigcup_{s \in R} K^{s}
$$

It follows from Proposition 1.1 that $K^{+\infty}$ consists of functions which can be extended as entire functions to $C^{n}$ and whose derivatives of all orders belong to $L^{2}$. It is useful to know that $K^{+\infty}$ is nonempty. As a matter of fact, we may state:

Proposition 1.3. If $\theta>0$, the function $x \rightarrow \exp \left(-\theta|x|^{2}\right)$ belongs to $K^{s}$ for all $s$ (hence to $K^{s, s^{\prime}}$ for all $s, s^{\prime}$ ). The space $K^{+\infty}$ is dense in $K^{s, s^{\prime}}$ for all $s, s^{\prime}$. 
Proof. The first part of the statement follows at once from the fact that the Fourier transform of $\exp \left(-\theta|x|^{2}\right)$ is the function

$$
(\pi / \theta)^{n / 2} \exp \left(-|\xi|^{2} / 4 \theta\right) \text {. }
$$

As for the second part, it follows from the fact that $(\operatorname{Exp} \cap \mathscr{S}) \subset K^{+\infty}$.

We recall that $\mathscr{E}^{\prime}$ denotes the space of distributions in $\boldsymbol{R}^{n}$ having a compact support.

Proposition 1.4. The space $\mathscr{E}^{\prime}$ is contained in $K^{s, s^{\prime}}$ for all $s<0$ and all $s^{\prime}$ real. The space $\mathscr{C}_{c}^{\infty}$ is dense in $K^{s, s^{\prime}}$ for all $s \leqq 0$ and all $s^{\prime}$ real.

Proof. That $\mathscr{E}^{\prime} \subset K^{s}$ for $s<0$ is an immediate consequence of the fact that the Fourier transform of a distribution with compact support is an analytic function in $\boldsymbol{R}_{n}$ which grows at infinity slower than some polynomial. As for the density of $\mathscr{C}_{c}^{\infty}$ in $K^{s, s^{\prime}}$ when $s \leqq 0$, it suffices to observe that $\mathscr{C}_{c}^{\infty}$ is dense in the Sobolev spaces $K^{0, s^{\prime}}=H^{s^{\prime}}$ and that the latter, or even simply $K^{00}=L^{2}$ is dense in every $K^{s}$ for $s<0$, as it contains $(\operatorname{Exp} \cap \mathscr{S})$.

Proposition 1.5. Let $s, s^{\prime}$ be arbitrary real numbers. Then $K^{-s,-s^{\prime}}$ can be canonically identified with the dual of $K^{s, s^{\prime}}$. The duality bracket between $K^{s, s^{\prime}}$ and $K^{-s,-s^{\prime}}$ is given by

$$
\langle u, v\rangle=(2 \pi)^{-n} \int\left(h^{s^{\prime}} e^{s h} \hat{u}\right)\left(h^{-s^{\prime}} e^{-s h} \hat{v}^{\smile}\right) d \xi,
$$

where $\hat{v}^{\vee}(\xi)=\hat{v}(-\xi)\left(u \in K^{s, s^{\prime}}, v \in K^{-s,-s^{\prime}}\right)$. The canonical antilinear isometry of $K^{s, s^{\prime}}$ onto its dual, $K^{-s,-s^{\prime}}$, is given by

$$
J_{s, s^{\prime}} u=\mathscr{F}^{-1}\left(h^{2 s^{\prime}}(\xi) e^{2 \operatorname{sh}(\xi)} \mathscr{F} \bar{u}\right) .
$$

Proof. Evident.

With the functions $h_{j}$ and $h$ are associated operators acting on elements $u \in K^{-\infty}$ :

$$
H_{j} u=\mathscr{F}^{-1}\left(h_{j} \hat{u}\right), \quad H u=\mathscr{F}^{-1}(h \hat{u}) .
$$

More generally, if $s$ and $s^{\prime}$ are real numbers, we may consider the operators defined by

$$
H^{s^{\prime}} u=\mathscr{F}^{-1}\left(h^{s^{\prime}} \hat{u}\right), \quad e^{s H} u=\mathscr{F}^{-1}\left(e^{s h} \hat{u}\right),
$$

which are endomorphisms, and in fact automorphisms, of $K^{-\infty}$. If $s_{1}$ and $s_{1}^{\prime}$ are real numbers, the operator $H^{s^{\prime}} e^{s H}$ induces an isometry of $K^{s_{1}}, s_{1}^{\prime}$ onto $K^{s_{1}-s, s_{1}^{\prime}-s^{\prime}}$.

As usual in this type of situation, convolution operators (acting on the spaces under study, here the $K^{s, s^{\prime}}$ ) are easy to deal with, whereas multipliers are more complicated. Consider a function $G$ in $\boldsymbol{R}_{n}$ such that, for some real numbers $a, a^{\prime}$,

$$
h^{a^{\prime}} e^{a h} G \in L^{\infty}\left(\boldsymbol{R}_{n}\right)
$$

Let then $g$ be the inverse Fourier transform of $G$ (this is well defined since $g \in K^{-\infty}$ ). 
We set, for any $u \in K^{s, s^{\prime}}, g * u=\mathscr{F}-1(G \mathscr{F} u)$, where $G \mathscr{F} u$ stands for the multiplicative product. It is obvious that $u \rightarrow g * u$ is a continuous linear map of $K^{s, s^{\prime}}$ into $K^{s+a, s^{\prime}+a^{\prime}}$. As a matter of fact, since $L^{\infty}$ makes up the set of all the multipliers of $L^{2}$, one obtains in the manner just described all the convolution operators from $K^{s, s^{\prime}}$ into $K^{s+a, s^{\prime}+a^{\prime}}$.

In particular, let $P(\xi)$ be a polynomial of degree $m$ on $\boldsymbol{R}_{n}$. The convolution of $u \in K^{s, s^{\prime}}$ with the inverse Fourier transform of $P(\xi)$ will be denoted by $P(-i \partial / \partial x) u$. This agrees with the standard notation when $u$ is a distribution. Clearly, $P(-i \partial / \partial x)$ defines a continuous linear map of $K^{s, s^{\prime}}$ into $K^{s, s^{\prime}-m}$.

We might mention that we could have studied the case where $s$ and $s^{\prime}$ are $n$-tuples of real numbers, $\left(s_{1}, \ldots, s_{n}\right),\left(s_{1}^{\prime}, \ldots, s_{n}^{\prime}\right)$ respectively, and defined the operators

$$
H^{s^{\prime}}=H_{1}^{s_{1}^{\prime}} \cdots H_{n^{n}}^{s^{\prime}}, \quad e^{\langle s, H\rangle}=e^{s_{1} H_{1}+\cdots+s_{n} H_{n}} .
$$

With such $n$-tuples are naturally associated spaces $K^{s, s^{\prime}}$. This approach is suited to situations where the different variables $x_{j}$ are given different rôles. We will not deal with such situations in this article.

2. About some members and multipliers of the space $K^{s}$. We keep the notation of $\S 1$. We assume momentarily that the dimension $n$ of the base space $\boldsymbol{R}^{\boldsymbol{n}}$ is equal to one (we continue to denote by $x, y$ the variables in it, by $\xi, \eta$ the ones in its dual $\left.\boldsymbol{R}_{n}\right)$. In this whole section, we write \|\| for the norm in $L^{2}\left(=L^{2}\left(\boldsymbol{R}^{n}\right)\right.$ or $\left.L^{2}\left(\boldsymbol{R}_{n}\right)\right)$; $R$ will be a number $>0$.

Proposition 2.1. The function $x \rightarrow(x+i R)^{-1}$ belongs to the space $K^{R,-1 / 2-\varepsilon}$ for all $\varepsilon>0$.

Proof. Indeed, the Fourier transform of the function under consideration is $-2 \pi i Y(\xi) e^{-R \xi}$, where $Y$ is the Heaviside function $(=1$ for $\xi>0$ and $=0$ for $\xi<0$ ).

COROLlaRY 1. The function $(x+i R)^{-1}$ belongs to $K^{s, s^{\prime}}$ for all $s<R$ and all $s^{\prime}$ real.

Proof. Combine Proposition 1.2 with Proposition 2.1.

CoRollary 2. The functions $\left(x^{2}+R^{2}\right)^{-1}$ and $x /\left(x^{2}+R^{2}\right)$ belong to $K^{s, s^{\prime}}$ for all $s<R$ and all $s^{\prime}$ real.

Proof. Just write

$$
\begin{aligned}
& \left(x^{2}+R^{2}\right)^{-1}=(2 i R)^{-1}\left\{(x-i R)^{-1}-(x+i R)^{-1}\right\} \\
& x /\left(x^{2}+R^{2}\right)=\frac{1}{2}\left\{(x-i R)^{-1}+(x+i R)^{-1}\right\}
\end{aligned}
$$

and apply Corollary 1 .

Proposition 2.2. If $|s|<R$, multiplication by $(x+i R)^{-1}$ defines a bounded linear operator of $K^{s}$, with norm $\leqq 2 \pi e^{|s|} /(R-|s|)$. 
Proof. By virtue of Proposition 1.3, it suffices to prove that multiplication by $(x+i R)^{-1}$ is a continuous map of $K^{+\infty}$, equipped with the norm of $K^{s}$, into $K^{s}$. Let $u \in K^{+\infty}$; the Fourier transform of $(x+i R)^{-1} u$ is

$$
F(\xi)=-2 i \pi \int_{0}^{+\infty} e^{-R \eta} \hat{u}(\xi-\eta) d \eta
$$

We have

$$
e^{s h(\xi)} F(\xi)=-2 i \pi \int_{0}^{+\infty} e^{k_{g}(\xi, \eta)} e^{-R \eta+|s| h(\eta)} e^{\operatorname{sh}(\xi-\eta)} \hat{u}(\xi-\eta) d \eta,
$$

where $k_{s}(\xi, \eta)=\operatorname{sh}(\xi)-|s| h(\eta)-s h(\xi-\eta)$ is $\leqq 0$ whatever $s, \xi, \eta$ real. We apply then Hölder's inequality

$$
\left\|e^{s h} F\right\| \leqq 2 \pi\left(\int_{0}^{+\infty} e^{-R \eta+|s| h(\eta)} d \eta\right)\left\|e^{s h} \hat{u}\right\|,
$$

whence easily the result.

COROLlaRY 1. If $|s|<R$, multiplication by $\left(x^{2}+R^{2}\right)^{-1}$ defines a bounded linear operator on $K^{s}$ with norm $\leqq\left(2 \pi e^{|s|} /(R-|s|)\right)^{2}$.

Proof. It suffices to write that $x^{2}+R^{2}=(x+i R)(x-i R)$.

COROLlaRY 2. If $|s|<R$, multiplication by $x /\left(x^{2}+R^{2}\right)$ defines a bounded linear operator on $K^{s}$ with norm $\leqq 2 \pi e^{|s|} /(R-|s|)$.

Proof. Apply (2.1).

Proposition 2.3. If $|s|<R$, multiplication by $\tan ^{-1}(x / R)$ defines a bounded linear operator on $K^{s}$.

Proof. First we assume $s \geqq 0$. The Fourier transform of $\tan ^{-1}(x / R)$ is the distribution

$$
U_{\xi}^{R}=-i \pi e^{-R|\xi|} p v(1 / \xi) .
$$

It is well known that $V=\mathscr{F}^{-1}(i \pi p v(1 / \xi))$ is a bounded function ( $\mathscr{C}^{\infty}$ in the complement of the origin). Let $\sigma$ be an arbitrary real number, $|\sigma|<R$. For $u \in K^{+\infty}$, consider

$$
e^{\sigma \xi}\left(U^{R} * \hat{u}\right)=\left(e^{\sigma \xi} U^{R}\right) *\left(e^{\sigma \xi} \hat{u}\right) .
$$

Set then $G_{R, \sigma}=\mathscr{F}^{-1}\left(e^{-R|\xi|+\sigma \xi}\right)$. We have, in virtue of Plancherel's identity,

$$
\left\|e^{\sigma \xi}\left(U^{R} * \hat{u}\right)\right\|=4 \pi^{2}\left\|\left(V * G_{R, \sigma}\right) \mathscr{F}-1\left(e^{\sigma \xi} \hat{u}\right)\right\|
$$

But by Hölder's inequality

$$
\left\|V * G_{R, \sigma}\right\|_{L^{\infty}} \leqq\|V\|_{L^{\infty}}\left\|G_{R, \sigma}\right\|_{L^{1}}
$$

and as a matter of fact, $G_{R, \sigma}(x)=(R / \pi)\left\{R^{2}+\left(x^{2}-i \sigma\right)\right\} \in L^{1}\left(R^{1}\right)$, so that

$$
\left\|G_{R, \sigma}\right\|_{L^{1}} \leqq R\left(R^{2}-\sigma^{2}\right)^{-1 / 2} .
$$


At any rate, we derive from (2.2)

$$
\left\|e^{\sigma \xi}\left(U^{R} * \hat{u}\right)\right\| \leqq C(R, \sigma)\left\|e^{\sigma \xi} \hat{u}\right\| \leqq C(R, \sigma)\left\|e^{|\sigma \xi|} \hat{u}\right\| .
$$

We apply this with $\sigma=s$ and $\sigma=-s$. We obtain:

$$
\left\|\cosh (s \xi)\left(U^{R} * \hat{u}\right)\right\| \leqq C(R, s)\left\|e^{s h} \hat{u}\right\| .
$$

But $e^{s h(\xi)} \leqq 2 e^{s} \cosh (s \xi)$, therefore

$$
\left\|e^{s h}\left(U^{R} * \hat{u}\right)\right\| \leqq 2 C(R, s) e^{s}\left\|e^{s h} \hat{u}\right\|,
$$

which is what we wanted to prove.

Suppose now that $s$ is $<0$. If $u, v$ are arbitrary elements of $K^{+\infty}$, we have (by Proposition 1.5)

$$
\left\langle u \tan ^{-1}(x / R), v\right\rangle=\left\langle u, v \tan ^{-1}(x / R)\right\rangle .
$$

This can be rephrased as follows: in the subspace $K^{+\infty}$ of $K^{s}$, the multiplication by $\tan ^{-1}(x / R)$ is the transpose of the same mapping in $K^{-s}$. Then the first part of the proof, together with Proposition 1.3, implies the sought result for $s<0$.

REMARK 2.1. It is not difficult to obtain an estimate for the norm of the mapping in $K^{s}$, "multiplication by $\tan ^{-1}(x / R)$ ". This norm is $\leqq$

$$
2 \pi^{2} R\left(R^{2}-s^{2}\right)^{-1 / 2}\|\mathscr{F}-1(p v 1 / \xi)\|_{L^{\infty}} .
$$

The preceding results have a natural extension to the case where the number $n$ of variables is arbitrary. The extension is trivial, due to the fact that $H=H_{1}+\cdots+H_{n}$ (see p. 7).

In the statement below, and also in the forthcoming ones, we use the following notation convention.

(2.3) Let $F$ be a function of a single variable, $x=\left(x_{1}, \ldots, x_{n}\right), p=\left(p_{1}, \ldots, p_{n}\right)$. We write

$$
F(x)^{p}=\left(F\left(x_{1}\right)\right)^{p_{1}} \cdots\left(F\left(x_{n}\right)\right)^{p_{n}} .
$$

Proposition 2.4. Let $R$ be a number $>0$, s a real number, $|s|<R$. There is a positive constant $C(R, s)$ such that, for all $n$-tuples $p, q, r$ multiplication by

$$
\left(\tan ^{-1}(x / R)\right)^{p}\left(R^{2}+x^{2}\right)^{-q}\left[x /\left(R^{2}+x^{2}\right)\right]^{r}
$$

is a bounded linear operator with norm $\leqq C(R, s)^{|p+q+r|}$.

REMARK 2.2. The constant $C(R, s)$ can be chosen so as to be a continuous function of $(R, s)$ in the angular domain $\left\{(s, R) \in R^{2} ;|s|<R\right\}$. This follows at once from the Corollaries of Proposition 2.2 and from Remark 2.1.

We may also combine Proposition 2.4 with Proposition 2.1 and its corollaries (extended to the case of $n$ variables):

Proposition 2.5. Let $d=\left(d_{1}, \ldots, d_{n}\right)$ be an $n$-tuple of integers $>0$. For all $s<R$, the space $K^{s}$ contains the function $\phi_{1}\left(x_{1}\right)^{d_{1}} \cdots \phi_{n}\left(x_{n}\right)^{d_{n}}$, where, for every $j=1, \ldots, n$, 
$\phi_{j}\left(x_{j}\right)$ is one of the following functions: $\left(x_{j}+i R\right)^{-1},\left(x_{j}-i R\right)^{-1},\left(x_{j}^{2}+R^{2}\right)^{-1}$, $x_{j} /\left(x_{j}^{2}+R^{2}\right)$.

3. Transformation of the "classical" situation. We consider the situation described at the beginning of the introduction and the problem (I)-(II). By possibly shrinking the open set $\Omega$, we may assume that the hypersurface $S$ is exactly the set of zeros in $\Omega$ of an analytic function whose gradient never vanishes in $\Omega$, and also that the following holds:

(3.1) The piece of hypersurface $S$ is noncharacteristic with respect to $\boldsymbol{P}$ at every one of its points.

We shall assume that the origin of $R^{n+1}$ lies on $S$ and that it is the point $x^{0}$ about which we study the problem (I)-(II).

In this section, we shall perform a succession of changes of variables and of functions which will ultimately transform our original problem (I)-(II) into a new one to which the theorems of $\$ 0$ can be applied.

FIRST CHANGE OF COORDINATES. Our first change of coordinates is standard practice; it might involve some shrinking of the set $\Omega$. The choice of the new coordinates, which we call $\left(y_{1}, \ldots, y_{n+1}\right)$, has a two-fold effect: it flattens the hypersurface $S$ which carries the Cauchy data, which is now defined, in $\Omega$, by the equation $y_{n+1}=0$; it puts the expression of the operator $\boldsymbol{P}$ (possibly after division by a nonvanishing function) into the form

$$
P\left(y, y_{n+1}, D_{y}, D_{y_{n+1}}\right)=D_{y_{n+1}}^{m}+\sum c_{p, p_{n+1}}\left(y, y_{n+1}\right) D_{y}^{p} D_{y_{n+1}}^{p_{n+1}} .
$$

In the right-hand side, the summation is performed over the $(n+1)$-tuples $\left(p, p_{n+1}\right)$ such that $|p|+p_{n+1} \leqq m, p_{n+1} \leqq m-1$ (we use systematically the notation $y=$ $\left.\left(y_{1}, \ldots, y_{n}\right), p=\left(p_{1}, \ldots, p_{n}\right)\right)$. It is clear that we have made full use of hypothesis (3.1). The coefficients $c_{p, p_{n+1}}$ in (3.2) are analytic functions in $\Omega$. More precisely, for a certain number $\kappa>0$,

(3.3) the coefficients $c_{p, p_{n+1}}\left(y, y_{n+1}\right)$ can be extended as holomorphic functions to the complex values of the variables $y_{j}(1 \leqq j \leqq n+1)$ in a neighborhood of the polydisk in $C^{n+1},\left|y_{j}\right| \leqq \kappa(1 \leqq j \leqq n+1)$.

SECOND CHANGE OF VARIABLES. This is given by

$$
y_{j}=x_{j}^{\prime}, \quad j=1, \ldots, n ; \quad y_{n+1}=\varepsilon t^{\prime}\left(\prod_{j=1}^{n} \cos ^{2}\left(x_{j}^{\prime} / \delta\right)\right),
$$

where $\varepsilon, \delta$ are numbers $>0$ which will be submitted to various conditions. Clearly (3.4) defines an analytic diffeomorphism of the set

$$
\left|y_{j}\right|<\delta \pi / 2, \quad j=1, \ldots, n ; \quad\left|y_{n+1}\right|<\varepsilon\left(\prod_{j=1}^{n} \cos ^{2}\left(y_{j} / \delta\right)\right)
$$

onto the set

$$
\left|x_{j}^{\prime}\right|<\delta \pi / 2, \quad j=1, \ldots, n ; \quad\left|t^{\prime}\right|<1
$$


We shall right away demand that $\varepsilon$ and $\delta$ be small enough so that the closure of the set (3.5) be contained in $\Omega$.

We must look quite carefully at the expression of $\boldsymbol{P}$ in the new coordinates $\left(x^{\prime}, t^{\prime}\right)$. First of all, we consider the coefficients of (3.2),

$$
c_{p, p_{n+1}}^{\prime}\left(x^{\prime}, t^{\prime}\right)=c_{p, p_{n+1}}\left(x^{\prime}, y_{n+1}\left(x^{\prime}, t^{\prime}\right)\right) \text {. }
$$

This much is obvious:

(3.7) Given any number $\delta>0$, if $\varepsilon$ is sufficiently small, the $c_{p, p_{n+1}}^{\prime}\left(x^{\prime}, t^{\prime}\right)$ can be extended as holomorphic functions in a neighborhood of the polydisk of $\mathrm{C}^{n+1}$, $\left|x_{j}^{\prime}\right| \leqq \kappa(1 \leqq j \leqq n),\left|t^{\prime}\right| \leqq 1$.

But we need to know more than just that! We observe that

$$
D_{y_{j}}=D_{x_{j}^{\prime}}+2 \frac{t^{\prime}}{\delta} \tan \left(x_{j}^{\prime} / \delta\right) D_{t}, \quad(1 \leqq j \leqq n), \quad D_{y_{n+1}}=\frac{1}{\varepsilon}\left(\prod_{j=1}^{n} \cos ^{2}\left(x_{j}^{\prime} / \delta\right)\right)^{-1} D_{t^{\prime}}
$$

As a matter of fact, we shall be interested in the differential operator

$$
\boldsymbol{Q}=\varepsilon^{m}\left\{\prod_{j=1}^{n} \cos ^{2}\left(x_{j}^{\prime} / \delta\right)\right\}^{m} \boldsymbol{P} .
$$

First of all, we observe (in view of (3.8)) that the coefficient of $D_{t^{\prime}}^{m}$ in the expression of $\boldsymbol{Q}$ in the coordinates $\left(x^{\prime}, t^{\prime}\right)$ is equal to

$$
\alpha\left(x^{\prime}, t^{\prime}\right)=P_{m}\left(x^{\prime}, \varepsilon t^{\prime}\left\{\prod_{j=1}^{n} \cos ^{2}\left(x_{j}^{\prime} / \delta\right)\right\}, \frac{\varepsilon}{\delta} t^{\prime} \theta, 1\right),
$$

where $P_{m}\left(y, y_{n+1}, D_{y}, D_{y_{n+1}}\right)$ is the principal part of (3.2) and $\theta$, the vector whose $j$ th component (for each $j=1, \ldots, n)$ is $\sin \left(2 x_{j}^{\prime} / \delta\right) \prod_{1 \leqq k \leqq n: k \neq j} \cos ^{2}\left(x_{k}^{\prime} / \delta\right)$. We derive, from (3.2) and (3.7):

(3.9) Given any $\delta>0$, if $\varepsilon$ is sufficiently small, $\alpha\left(x^{\prime}, t^{\prime}\right)$ can be extended as a nevervanishing holomorphic function in a neighborhood of the polydisk in $C^{n+1},\left|x_{j}^{\prime}\right| \leqq \kappa$ $(j=1, \ldots, n),\left|t^{\prime}\right| \leqq 1$.

We need also some information about the remaining coefficients in the expression of $Q$. This is a linear combination, with the functions $c_{p, p_{n+1}}^{\prime}\left(x^{\prime}, t^{\prime}\right)$ as coefficients, of the differential operators

$$
\varepsilon^{m-p_{n+1}}\left\{\prod_{j=1}^{n} \cos ^{2}\left(x_{j}^{\prime} / \delta\right)\right\}^{m-p_{n+1}} D_{t^{p_{n+1}}}^{p^{\prime}}\left\{\prod_{k=1}^{n}\left(D_{x_{k}^{\prime}}+\frac{2 t^{\prime}}{\delta} \tan \left(x_{k}^{\prime} / \delta\right) D_{t^{\prime}}\right)^{p_{k}}\right\}
$$

where $|p|+p_{n+1} \leqq m$ (we shall now only look at the case where $p_{n+1}<m$ ). Suppose for a moment that $x$ stands for a single real variable and $p$ for some integer $>0$, and consider the operator

$$
\varepsilon^{p}\left(\cos ^{2}(x / \delta)\right)^{p}\left(D_{x}+2 \delta^{-1} \tan (x / \delta) t^{\prime} D_{t^{\prime}}\right)^{p} .
$$

We apply the following easy lemma of algebra (its proof is left to the student): 
Lemma 3.1. Let $A, B$ be two elements in some ring $\mathscr{R}$, such that $A$ and $[A, B]=A B$ $-B A$ commute. Then, given any integer $p>0, A^{p} B^{p}$ belongs to the subring spanned by $A B$ and $[A, B]$.

We apply the lemma with the choices

$$
A=\varepsilon \cos ^{2}(x / \delta), \quad B=D_{x}+2 \delta^{-1} \tan (x / \delta) t^{\prime} D_{t^{\prime}} .
$$

Note that we have

$$
A B=\frac{\varepsilon}{\delta} \delta \cos ^{2}(x / \delta) D_{x}+\frac{\varepsilon}{\delta} \sin (2 x / \delta) t^{\prime} D_{t^{\prime}}, \quad[A, B]=\frac{\varepsilon}{\delta} \sin (2 x / \delta) .
$$

The operator (3.11) belongs therefore to the ring generated by these two differential operators. Returning to (3.10) we see that it belongs to the algebra spanned by the differential operators

$$
\delta \cos ^{2}\left(x_{j}^{\prime} / \delta\right) D_{x_{j}^{\prime}} \quad(j=1, \ldots, n), \quad D_{t^{\prime}},
$$

over the algebra spanned by the functions $\sin \left(x_{j}^{\prime} / \delta\right), \cos \left(x_{j}^{\prime} / \delta\right), t^{\prime}$ and by $\varepsilon$ and $\varepsilon / \delta$. We may write the expression of $\alpha^{-1} Q$ in the coordinates $\left(x^{\prime}, t^{\prime}\right)$ :

$$
\begin{aligned}
& \alpha^{-1}\left(x^{\prime}, t^{\prime}\right) Q\left(x^{\prime}, t^{\prime}, D_{x^{\prime}}, D_{t^{\prime}}\right) \\
& =D_{t^{\prime}}^{m}+\sum_{p, l} b_{p, l}\left(x^{\prime}, t^{\prime}\right)\left\{\prod_{j=1}^{n}\left(\delta \cos ^{2}\left(x_{j}^{\prime} / \delta\right) D_{x_{j}}\right)^{p_{j}}\right\} D_{t^{\prime}}^{l} .
\end{aligned}
$$

The summation with respect to $p, l$ is performed according to the customary rule $|p|+l \leqq m, l<m$. In virtue of (3.7) and (3.9), we may state:

(3.13) Given any $\delta>0$, if $\varepsilon$ is sufficiently small, the coefficients $b_{p, l}\left(x^{\prime}, t^{\prime}\right)$ can be extended as holomorphic functions in a neighborhood of the polydisk $C^{n+1},\left|x_{j}^{\prime}\right| \leqq \kappa$ $(j=1, \ldots, n),\left|t^{\prime}\right| \leqq 1$.

THIRD CHANGE OF VARIABLES. This will be our last change of variables. Its effect is to "globalize" the situation with respect to the $x$-variables. It is defined by

$$
x_{j}=R \tan \left(x_{j}^{\prime} / \delta\right), \quad j=1, \ldots, n ; t=t^{\prime},
$$

where $R$ is some number $>0$ (its choice will depend on the applications we are interested in, and will determine the choice of $\delta$ and therefore, in view of the previous requirements, the one of $\varepsilon$ ). At any rate, (3.14) defines an analytic diffeomorphism of the set (3.6) onto the strip $\left\{(x, t) \in \boldsymbol{R}^{n+1} ;|t|<1\right\}$. We have

$$
R D_{x_{j}}=\delta \cos ^{2}\left(x_{j}^{\prime} / \delta\right) D_{x_{j}^{\prime}} \quad(1 \leqq j \leqq n) .
$$

In view of (3.12), the differential operator $\alpha^{-1} Q$ is transformed by (3.14) into a differential operator $l$ on the strip $|t|<1$ of $R^{n+1}$ whose expression is given by:

$$
L\left(x, t, D_{x}, D_{t}\right)=D_{t}^{m}+\sum R^{|p|} b_{p, l}\left(\delta \tan ^{-1}(x / R), t\right) D_{x}^{p} D_{t}^{l},
$$

with summation rule, $|p|+l \leqq m, l<m$. 
The original problem (I)-(II) (see Introduction) has been finally transformed into the new one:

$$
\begin{aligned}
L\left(x, t, D_{x}, D_{t}\right) U(x, t) & =F(x, t), \\
\left.D_{t}^{k} U(x, t)\right|_{t=0} & =G_{k}(x), \quad k=0, \ldots, m-1 .
\end{aligned}
$$

We have set

$$
\begin{aligned}
F(x, t) & =\varepsilon^{m}\left\{\prod_{j=1}^{n} \frac{R^{2}}{R^{2}+x_{j}^{2}}\right\}^{m}\left(\alpha^{-1} f\right)\left(\delta \tan ^{-1}(x / R), t\right), \\
G_{k}(x) & =\varepsilon^{k}\left\{\prod_{j=1}^{n} \frac{R^{2}}{R^{2}+x_{j}^{2}}\right\}^{k} g_{k}\left(\delta \tan ^{-1}(x / R)\right), \quad k=0, \ldots, m-1 .
\end{aligned}
$$

Giving a meaning to (3.17) and (3.18) might raise some delicate questions, for $f$ and the $g_{k}$ 's may not be ordinary objects like functions or distributions and the sense of the substitutions (3.14) may need some clarification. In fact, (3.17) and (3.18) might be taken as definitions of $f$ and $g_{k}$. In the applications, we shall ask that $F(x, t)$ and $G_{k}(x)$ take their values in spaces $K^{s, s^{\prime}}$ (with respect to the variables $x$ ), and this will define what $f$ and $g_{k}$ must be. It should be said, however, that when $F$ and $G_{k}$ are functions or distributions, the same will be true of $f$ and $g_{k}$ (when they are distributions but not functions, the substitutions (3.14) must be defined as extensions of the same performed on functions).

We go back to equations (3.15) and (3.16). We propose to transform them into an ordinary differential equation, with prescribed datum at $t=0$, of the type considered in $\$ 0$. We do the transformation according to standard rules. At first we proceed purely formally. We set, for $k=1, \ldots, m$,

$$
U_{k}=H^{m-k} D_{t}^{k-1} U
$$

Observe that we have, for $k=1, \ldots, m-1$,

$$
D_{t} U_{k}=H U_{k+1} \text {. }
$$

As for equation (3.15), it becomes

$$
D_{t} U_{m}=\sum_{p, l} R^{|p|} b_{p, l}\left(\delta \tan ^{-1}(x / R), t\right)\left(D_{x}^{p} H^{-m+l}\right) H U_{l+1}+F .
$$

As one does usually, we introduce the $m \times m$ matrix

$$
\left(\begin{array}{rrrrrr}
0 & 1 & 0 & \cdots & 0 & 0 \\
0 & 0 & 1 & \cdots & 0 & 0 \\
. & . & . & \cdots & . & . \\
0 & 0 & 0 & \cdots & 0 & 1 \\
-\beta_{1} & -\beta_{2} & -\beta_{3} & \cdots & -\beta_{m-1} & \beta_{m}
\end{array}\right)
$$

where we have set

$$
\beta_{k}=\sum_{|p| \leqq m-k+1} R^{|p|} b_{p, k-1}\left(\delta \tan ^{-1}(x / R), t\right)\left(D_{x}^{p} H^{-m+k-1}\right) .
$$


Let then $E$ be an $m$-dimensional complex vector space where a basis has been given (in other words, $E=C^{m}$ ), equipped with the Hilbert space structure defined by this basis. Let us set $\mathbf{u}(t)=\left(U_{1}, \ldots, U_{m}\right)$, regarding $\mathbf{u}(t)$ as a function of $t$ in $[-1,+1]$ with values in a certain space $\mathscr{K}$ of vector valued (the vectors belonging to $E$ ) functions (or distributions, or "ultradistributions") with respect to the variables $x$. We shall assume that the matrix (3.23) defines a linear operator on this space $\mathscr{K}$, which we denote by $B(t)$.

We also set $\mathbf{f}(t)=(0, \ldots, 0, \mathbf{F})$.

Finally, equations (3.21)-(3.22) become

$$
D_{t} \mathbf{u}(t)-B(t) H \mathbf{u}(t)=\mathbf{f}(t),
$$

whereas the condition at time $t=0$ becomes

$$
\mathbf{u}(0)=\mathbf{u}_{0},
$$

setting

$$
\mathbf{u}_{0}=\left(H^{m-1} G_{0}, H^{m-2} G_{1}, \ldots, G_{m-1}\right),
$$

and observing that, in view of (3.20), (3.16) can be rewritten as

$$
\left.U_{k}\right|_{t=0}=H^{m-k} G_{k-1} \quad(k=1, \ldots, m) .
$$

We have thus obtained a system of two equations, namely (3.25)-(3.26), which looks like equations (0.5)-(0.6). It is now time to give a precise meaning to (3.25)(3.26), which will make this similarity rigorous.

4. On solvability of linear PDE's with analytic coefficients. Equations (3.15)(3.16) have been converted into the system (3.25)-(3.26), to which we want to apply the methods of $\$ 0$. For this we shall require that the data, $f(t)$ and $u_{0}$, as well as the solution $\mathbf{u}(t)$, take their values in some tensor product space $K^{s} \otimes E$. Here, just as in $\S 3, E$ is a finite dimensional complex Hilbert space; $K^{s} \otimes E$, or more generally $K^{s, s^{\prime}} \otimes E$, carries the tensor product Hilbert space structure. An element of $K^{s, s^{\prime}} \otimes E$ is, vaguely speaking, a "vector" whose coordinates, in any given basis of $E$, are elements of $K^{s, s^{\prime}}$. The norm and the inner product in $K^{s, s^{\prime}} \otimes E$ will be denoted just as they were when no $\otimes E$ was present: \|\|$_{s, s^{\prime}}$ and $(,)_{s, s^{\prime}}$. Any linear operator acting on $K^{s, s^{\prime}}$ will be made to act on the tensor product $K^{s, s^{\prime}} \otimes E$ by acting on the first factor, $K^{s, s^{\prime}}$, and inducing the identity mapping on the second one, $E$. Thus, for $a$ and $a^{\prime}$ real numbers, $e^{a H}$ and $H^{a^{\prime}}$ act on $K^{s, s^{\prime}} \otimes E$ (and map isometrically this space onto $K^{s-a . s^{\prime}-a^{\prime}} \otimes E$ ).

We shall then apply the results of $\$ 0$, choosing, as one-parameter family of Banach spaces $E_{s}$, the spaces $K^{s} \otimes E$, and as operator $\bigwedge$, the operator $H$. Conditions (0.1) and (0.2) are easily checked. In order to see that the norm of the map $H: K^{s} \otimes E \rightarrow K^{s^{\prime}} \otimes E\left(s>s^{\prime}\right)$ is $\leqq e^{-1}\left(s-s^{\prime}\right)^{-1}$ (and, in fact, is equal to this number), one performs a Fourier transformation and uses the fact that, for $\lambda \geqq 0$, $\lambda e^{-\lambda} \leqq e^{-1}$. 
In accordance with this, we require that our data $\mathbf{u}_{0}$ and $\mathbf{f}(t)$ satisfy conditions $(0.7),(0.8)$. Observe that $(0.7)$ means

$$
\mathbf{u}_{0} \in K^{s_{0}, 1} \otimes E \text {. }
$$

Going back to the definition (3.27) of $\mathbf{u}_{0}$, we see that (4.1) means

$$
G_{k} \in K^{s_{0}, m-k}, \quad k=0, \ldots, m-1 .
$$

As for the solution $\mathbf{u}(t)$, it will have to be a continuously differentiable function of $t$ with values in $K^{s} \otimes E\left(s<s_{0}\right)$. This is equivalent with saying (cf. (3.20)) that

(4.3) for every $k=1, \ldots, m, D_{t}^{k} U$ is a continuous function of $t$ with values in $K^{s, m-k}$.

As $s<s_{0}$ is arbitrary, trivially this will also be true for $k=0$ :

(4.4) $U$ is a continuous function of $t$ with values in $K^{s, m}$.

When we extend $t$ into the complex domain, "continuous" and "continuously differentiable", in the above statements, and also in the following ones, must be replaced by "holomorphic".

It remains to check that equation (3.25) makes sense, i.e., that $B(t)$ operates in the way we want, and satisfies $(0.3)$ and $(0.4)$. In order to prove this, we must now choose the parameters $R, \delta, \varepsilon$ in (3.4) and (3.14).

Choice of the PARAmeters. We suppose that we are given $s_{0}$, an arbitrary real number. Then we choose for $R$ any number such that $\left|s_{0}\right|<R$. Then the number $\sigma$ of $\$ 0$ might be any positive number such that

$$
\left|s_{0}\right| \leqq \sigma<R .
$$

The choice of $\delta$ will then follow from the one of $R$ and $\sigma$, in the following manner. We apply Proposition 2.4 and we observe that the multipliers $\tan ^{-1}\left(x_{j} / R\right)$, $j=1, \ldots, n$, define bounded linear operators of $K^{s}$ into itself, for all real $s,|s| \leqq \sigma$, with norm $\leqq M$, a number which depends only on $R$ and $\sigma$. We shall then require

$$
\delta M \leqq \kappa, \text { the number in (3.3). }
$$

This enables us to choose $\delta>0$. Once we have done it, we choose $\varepsilon>0$ so that (3.7), (3.9) and (3.13) be satisfied.

The stage is now set for the application of Theorems 0.1 and 0.2 . Indeed, returning to the expression (3.23) of $B(t)$, we look at the ones of the entries $\beta_{k}$ given in (3.24), and, in fact, at the individual terms

$$
R^{|p|} b_{p, k-1}\left(\delta \tan ^{-1}(x / R), t\right)\left(D_{x}^{p} H^{-m+k-1}\right) .
$$

Since $|p| \leqq m-k+1, D^{p} H^{-m+k-1}$ defines a bounded linear operator of $K^{\lambda, \lambda^{\prime}}$ whatever be the real numbers $\lambda, \lambda^{\prime}$, with norm bounded independently of $\lambda, \lambda^{\prime}$. As for the multipliers $b_{p, k-1}\left(\delta \tan ^{-1}(x / R), t\right)$, we combine our choice (4.6) with property (3.13). We conclude easily that

(4.7) $B(t)$ is a holomorphic mapping of a neighborhood of the closed unit disk in the complex plane (of the variable $t$ ) with values in the Banach space of bounded linear 
operators of $K^{s} \otimes E$ into itself, for all real $s,|s| \leqq \sigma$, with norm bounded by a number $\tau>0$, independent of $t$ and $s$.

Clearly, this implies at once (0.3) and (0.4) (with $\rho=1$ ).

Finally we obtain, by applying Theorems 0.1 and 0.2 :

THEOREM 4.1. Let $F(x, t)$ be a continuous function of $t,|t| \leqq 1$, with values in the space $K^{s_{0}}$ relative to the variables $x$, and if $t$ is complex, holomorphic for $|t|<1$ with values in $K^{s_{0}}$. Consider $m$ Cauchy data $G_{k} \in K^{s_{0}, m-k}(0 \leqq k<m)$. Let $s$ be any real number such that $s_{0}-\tau<s<s_{0}$ and $|s|<\sigma$.

There is a unique function $U(x, t)$ with the following property:

(4.8) for each $k=0,1, \ldots, m, D_{t}^{k} U(x, t)$ is a continuous function of $t$, holomorphic if $t$ is a complex variable, in the set defined by $|t|<\tau^{-1}\left(s_{0}-s\right)$, with values in $K^{s, m-k}$, and verifying:

$$
\begin{aligned}
& L\left(x, t, D_{x}, D_{t}\right) U(x, t)=F(x, t) \text { for }|t|<\tau^{-1}\left(s_{0}-s\right), \\
& \left.D_{t}^{k} U(x, t)\right|_{t=0}=G_{k}(x) \quad \text { for every } k=0, \ldots, m-1 .
\end{aligned}
$$

The "real" version of Theorem 4.1 (i.e., the version in which $t$ is regarded as a real variable) yields a weak solvability result for linear PDE's with analytic coefficients, as we have pointed out in the introduction. If then we work our way backwards through the (analytic) diffeomorphisms of $\S 3$, we see that $U(x, t)$ (resp., $F(x, t)$, resp., $G_{k}(x)$ ) is a function-or a distribution (in some strip $\left.|t|<\eta\right)$, if and only if the solution $u$ (resp. the right-hand side $f$, resp., the Cauchy datum $g_{k}$ ) has the same property (in the preimage of the strip $|t|<\eta$ ). In a sense, this establishes an equivalence between the "solvability problem" relative to the differential operator $\boldsymbol{P}$ and the same problem relative to $L\left(x, t, D_{x}, D_{t}\right)$.

5. Derivation of the classical theorems. We begin by proving Holmgren's uniqueness theorem.

As before (cf. §3), we consider a differential operator $\boldsymbol{P}$ of order $\boldsymbol{m}$ in the open neighborhood of the origin, $\Omega$, in $\boldsymbol{R}^{n+1}$; the coefficients of $\boldsymbol{P}$ are complex valued (real) analytic functions in $\Omega$. We are given a piece of hypersurface $S_{1}$ in $\Omega$, of class $\mathscr{C}^{1}$, passing through the origin, and noncharacteristic with respect to $\boldsymbol{P}$ at that point. We shall suppose that the complement of $S_{1}$ with respect to $\Omega$ consists of two connected open sets, $\Omega_{1}^{+}$and $\Omega_{1}^{-}$. We recall the classical form of Holmgren's theorem $\left({ }^{3}\right)$ :

THEOREM 5.1 (HOLMGREN). Under the previous assumptions, there is an open neighborhood $\mathcal{O}_{1}$ of 0 in $\Omega$ such that, for all $\mathscr{C}^{m}$ functions $u$ in $\Omega$, verifying

$$
\boldsymbol{P} u=0 \text { in } \Omega, \quad u=0 \text { in } \Omega_{1}^{+},
$$

we have necessarily $u=0$ in $\mathcal{O}_{1}$.

( $\left.{ }^{3}\right)$ The "modern" form of Holmgren's theorem, proved in Hörmander [1] (Theorem 5.3.1), can easily be derived from a variant of Theorem 0.1 . See Treves [4]. 
Proof. Possibly after shrinking $\Omega$, we may find a piece of analytic hypersurface in $\Omega, S$, with the properties listed in the initial paragraphs of $\S 3: S$ is noncharacteristic with respect to $\boldsymbol{P}$ at every one of its points; $S$ contains the origin, and its complement with respect to $\Omega$ consists of two connected open sets, $\Omega^{+}$and $\Omega^{-}$; $S_{1}$ lies entirely on one side of $S$, say $S_{1} \subset \bar{\Omega}^{-}$, i.e., $\Omega_{1}^{-} \subset \Omega^{-}$; the intersection of $S$ and $S_{1}$ consists solely of the origin.

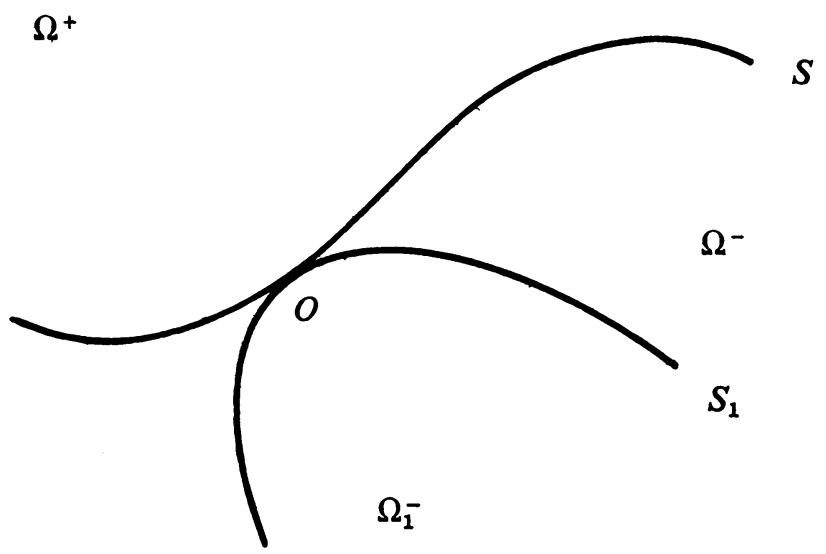

FIGURE 1

We perform the changes of variables described in $\S 3$. They transform property (5.1) into

$$
\begin{aligned}
L\left(x, t, D_{x}, D_{t}\right) U(x, t) & =0 \text { for }|t|<1, \\
U(x, t) & =0 \text { for } 0<t<1 \text { and all } x \in R^{n} .
\end{aligned}
$$

Furthermore, we know that $U(x, t)$ is a $\mathscr{C}^{m}$ function of $(x, t)$ in the strip $|t|<1$. We know also that

$$
t \mapsto U(x, t)
$$

is a $\mathscr{C}^{m}$ function of $t,|t|<1$, with values in the space of continuous functions with compact support in $\boldsymbol{R}^{n}$. This is seen at once on Figure 1. It suffices then to apply Proposition 1.4 and the uniqueness part of Theorem 4.1.

We come now to the classical Cauchy-Kovalevska theorem. In order to prove it, we go back to the original problem (I)-(II). It is convenient, and customary, to extend all the intervening functions to the complex domain: we may assume that $\Omega$ is the real part of an open neighborhood of $O$ in the complex space $C^{n}, \Omega^{C}$, in which we are given a complex analytic submanifold of codimension $1, S^{C}$, whose intersection with $\Omega$ (i.e., with the real space) is equal to $S$. We are also given a differential operator in the complex sense (that is, involving only the $\partial / \partial z_{j}$ and no $\left.\partial / \partial \bar{z}_{j}\right)$, with holomorphic coefficients in $\Omega^{C}, \boldsymbol{P} \boldsymbol{C}$, which induces the real differential 
operator $\boldsymbol{P}$ in $\Omega$ (by taking the $z_{j}$ real). We may also extend the hypothesis (3.1) by assuming now:

(5.4) The submanifold $S^{C}$ is noncharacteristic with respect to $P C$ at every one of its points.

Here, now, is the usual version of the Cauchy-Kovalevska theorem:

THEOREM 5.2. If (5.4) holds, there is an open neighborhood $O^{C}$ of $O$ in $C^{n}$ such that the following is true:

To every holomorphic function $f$ in $\Omega^{C}$ and to every m-tuple $\left(g_{0}, \ldots, g_{m-1}\right)$ of holomorphic functions in $S^{C}$, there is a unique holomorphic function $u$ in $\mathcal{O C}^{C}$ such that

(5.5) $P c_{u}=f$ in $\mathcal{O C}$,

(5.6) $(\partial / \partial v)^{k} u=g_{k}$ in $O^{C} \cap S^{C}$ for $k=0, \ldots, m-1$.

The normal derivative $\partial / \partial \nu$ is to be understood, here, in the complex sense, recalling that $S^{C}$ is a submanifold of codimension 1 of $\Omega^{c}$.

Proof of Theorem 5.2. By possibly shrinking $\Omega^{C}$, we may find a holomorphic function $\phi$ in this open set, which satisfies $(\partial / \partial v)^{k} \phi=g_{k}$ on $\Omega^{C} \cap S^{C}$ for all $k=0, \ldots, m-1$. Then, (5.5) and (5.6) can be rewritten into

(5.7) $P^{C}(u-\phi)=f-P^{C} \phi$ in $\mathcal{O C}$,

(5.8) $(\partial / \partial v)^{k}(u-\phi)=0$ in $\mathcal{O}^{C} \cap S^{C}$ for $k=0, \ldots, m-1$.

In other words, it suffices to prove the existence and uniqueness of the solution $u$ when the Cauchy data $g_{k}$ are all identically equal to zero. That is what we suppose from now on.

We perform (in real space) the changes of variables of §3. They lead to the equations

$$
\begin{aligned}
L\left(x, t, D_{x}, D_{t}\right) U(x, t) & =F(x, t), \\
\left.D_{t}^{k} U(x, t)\right|_{t=0} & =0 \text { for } k=0, \ldots, m-1 .
\end{aligned}
$$

Let us return to the expression (3.17) of $F$ in terms of $f$. In view of the hypotheses about $f$, and of (3.9), if $\kappa>0$ is small enough, $\left(\alpha^{-1} f\right)\left(x^{\prime}, t\right)$ can be extended as a holomorphic function in a neighborhood of the polydisk of $C^{n+1},\left|x_{j}^{\prime}\right| \leqq \kappa(1 \leqq j \leqq n)$, $|t| \leqq 1$. We make then the choices described in p. 16. In particular, because of (4.6) and by the same reasoning as the one used in p. 16, we see that the multiplier $\left(\alpha^{-1} f\right)\left(\delta \tan ^{-1}(x / R), t\right)$ defines a holomorphic mapping of a neighborhood of the closed unit disk $|t| \leqq 1$ in $C^{1}$, into the Banach space of bounded linear operators of $K^{\sigma}$ into itself. Assuming that $m$ is $>0$ (otherwise the theorem to be proved is trivial), we derive from Proposition 2.5 that

$$
\left\{\prod_{j=1}^{n} \frac{R^{2}}{R^{2}+x_{j}^{2}}\right\}^{m}
$$

belongs to $K^{\sigma}$. Therefore, $F(x, t)$ is a holomorphic function in a neighborhood of $\left\{t \in C^{1} ;|t| \leqq 1\right\}$ with values in $K^{\sigma}$. We apply the complex version of Theorem 4.1 
(with $s_{0}=\sigma>0$ ). Choosing $0<s<s_{0}$, we conclude that there is a unique solution $U(x, t)$ of (5.9)-(5.10) which is a holomorphic function of $t,|t|<\tau^{-1}(\sigma-s)$, with values in $K^{s}$. Since $s$ is $>0$, we may apply Proposition $1.1: U(x, t)$ is a holomorphic function of $(x, t)$ in the open subset of $C^{n+1}$ defined by the inequalities

$$
\left|\operatorname{Im} x_{j}\right|<s, j=1, \ldots, n, \quad|t|<\tau^{-1}(\sigma-s) .
$$

Reverting to the solution $u$ of (5.5)-(5.6) via the (inverse) changes of variables of $\$ 3$ completes the proof of Theorem 5.2.

\section{REFERENCES}

1. L. Hörmander, Linear partial differential operators, 3rd ed., Springer-Verlag, Berlin, 1965.

2. L. Nirenberg and F. Treves, On solvability of first order linear partial differential equations, Comm. Pure Appl. Math. 14 (1963), 331-351.

3. L. V. Ovsyannikov, A singular operator in a scale of Banach spaces, Dokl. Akad. Nauk SSSR 163 (1965), 819-822 = Soviet Math. Dokl. 6 (1965), 1025-1028.

4. F. Treves, Ovsyannikov theorem and hyperdifferential operators, I.M.P.A., Rio-de-Janiero, 1968.

Purdue University,

LAFAYETTE, INDIANA 